\title{
Sistema de Recomendação de Tópicos e Recursos Educacionais Utilizando Redes Sociais
}

\author{
João Pedro Batista Ferreira, Renata Lopes Rosa, Demóstenes Zegarra Rodríguez \\ Departamento de Ciência da Computação - Universidade Federal de Lavras (UFLA) \\ CEP 37200-000 - Lavras - MG - Brazil \\ jpbferreira@computacao.ufla.br , \{renata.rosa, demostenes.zegarra\}@ \\ dcc.ufla.br
}

\begin{abstract}
Social networks provide current and internationally relevant content that can help in the creation and modernization of contents offered in the classroom. In this context, we propose a recommendation system that suggests teaching topics and educational tools to the teacher. The analysis of these topics is based on the comments of the users of the social network. An evaluation metric is implemented, considering positive texts by sentiment analysis and after other parameters: amount of likes, reactions and share posts. The sentiment analysis performed uses a word dictionary built specifically for the area of education, allowing you to determine the overall sentiment intensity of a sentence.
\end{abstract}

Resumo. As redes sociais disponibilizam conteúdos atuais e de relevância internacional que podem auxiliar na criação e modernização de conteúdos ofertados em sala de aula. Nesse contexto, propomos um sistema de recomendação que sugira tópicos de ensino e ferramentas educacionais ao docente. A análise destes tópicos está baseada nos comentários dos usuários da rede social. Uma métrica de avaliação é implementada, considerando textos positivos extraídos da análise de sentimentos e posteriormente outros parâmetros: quantidade de curtidas, reações e compartilhamentos de posts. A análise de sentimentos realizada utiliza um dicionário de palavras construído especificamente para a área de educação, permitindo determinar a intensidade de sentimento global de uma frase.

\section{Introdução}

$\mathrm{O}$ aumento na facilidade de acesso à Internet possibilita o crescimento cada vez maior do número de usuários nas diversas redes sociais, viabilizando o acesso à informação e ao conhecimento disponível na Internet. Com base nessa observação, vê-se presente a criação de jogos interativos, vídeos informativos, textos e imagens que vêm sendo explorados por diversas instituições ou grupos na disseminação de novos conhecimentos, conceitos e na internacionalização da informação.

Atualmente, as pessoas comumente expressam seus sentimentos e opiniões por meio das redes sociais e micro-blogs. Portanto, existem muitas ferramentas de análise de sentimentos (AS) para textos extraídos de micro-blogs, mas a maioria dessas ferramentas, tais como ANEW, OpinionFinder, SentiWordNet possuem um dicionário genérico não voltado a educação e ensino. 
O presente trabalho visa extrair informações das páginas, CS50 e Codecademy, da rede social Facebook. Tais páginas selecionadas possuem um caráter educacional na área de computação e programação, com o intuito de analisar os conteúdos com maior disseminação e cujo texto, vídeo ou imagem possuam um sentimento global positivo, do ponto de vista educacional. No caso de vídeos e imagens, são analisados os nomes descritivos de tais conteúdos. Utilizando a ferramenta de extração de dados Netvizz, é possível extrair, por exemplo, a quantidade de curtidas (ou likes), reações (emoções expressas por meio de figuras) e compartilhamento dos posts de uma página, além do número de usuários por país que curtem ou seguem a página. Posteriormente, tais conteúdos passam por um dicionário de palavras para extrair o sentimento da frase, vídeo ou imagem. Destacamos que um dicionário voltado a educação é implementado, contendo palavras associadas a ensino, informação, aulas, entre outras. Efetuando a análise de sentimentos para extração de textos positivos e posteriormente considerando parâmetros de medição de disseminação de posts, implementamos uma métrica de avaliação a ser aplicada em um sistema de recomendação (SR). Tal SR pretende sugerir os conteúdos com maior disseminação aos docentes cadastrados, de polaridade positiva e com foco em educação.

\section{Trabalhos Relacionados}

A análise de sentimentos (AS) tem como objetivo extrair as opiniões das pessoas, por meio da análise da polaridade (positiva, negativa ou neutra) de um texto [Pang et al., 2008]. A AS pode ser efetuada por meio de aprendizagem de máquina ou por um dicionário léxico, no qual cada palavra está associada a um número em uma escala de -5 a +5 (mais negativo para mais positivo). Após a classificação de intensidade de sentimento global de um texto, a AS pode ser aplicada em um SR para poder sugerir conteúdos de acordo com a polaridade dos textos analisados. Estudos (Pereira et al., 2015a) (Almeida et al., 2015) vêm utilizando as informações das redes sociais para recomendar conteúdos de cunho educacional. Porém o SR proposto se diferencia de tais trabalhos por sugerir conteúdos educacionais, extraídos das redes sociais, utilizando uma métrica de avaliação, considerando AS e outros parâmetros: quantidade de curtidas, reações e compartilhamentos de posts.

\section{Proposta de Sistema de Recomendação para o Ensino}

A metodologia utilizada para a construção do SR voltado à área educacional inclui as seguintes etapas:

- Extração de posts da rede social Facebook, das páginas CS50 e Codecademy. A extração dos dados é efetuada por meio de um aplicativo denominado Netvizz.

- Análise de sentimentos dos textos resultantes da fase anterior para extração da polaridade dos textos, cujo dicionário próprio para educação possui palavras relacionadas à educação, tais como "estudo", "aulas", "educativo" e com pontuações positivas, variando de $+2 \mathrm{a}+4$, para tais palavras.

- Extração da quantidade de curtidas, reações e compartilhamentos dos posts extraídos por meio de um script e aplicação da métrica de avaliação.

- Desenvolvimento de um protótipo de SR, recomendando conteúdos que possibilitem agregar informações em conteúdos didáticos de docentes da área de computação. Foram feitas duas sugestões, uma utilizando a métrica de avaliação e outra sem a métrica para fim de comparação. Testes prévios foram efetuados com 45 docentes da área de computação. 
VI Congresso Brasileiro de Informática na Educação (CBIE 2017)

Anais do XXVIII Simpósio Brasileiro de Informática na Educação (SBIE 2017)

\subsection{Métrica de Avaliação com uso de Parâmetros de Medição de Disseminação de Conteúdo}

A métrica de avaliação abaixo foi utilizada do SR implementado. Somente os textos positivos, filtrados pela AS, foram considerados na métrica abaixo, os demais foram descartados. Segundo análise experimental os posts com maior compartilhamento possuíam uma reação extremamente positiva (LOVE) acima de 10, e nesse caso a soma do número de curtidas, mais as reações em geral (LOVE, WOW, HAHA, SAD, ANGRY e THANKFUL) e mais o compartilhamento possuíam um valor mínimo de 150 (curtidas + compartilhamento + reações). Portanto, quando a métrica de avaliação (Métrica_avaliação_textos_positivos) possui valores acima de 150 então o conteúdo é sugerido pelo SR ao docente.

\section{Métrica_avaliação_textos_positivos = numero_curtidas + numero_reações + numero_compartilhamento}

\section{Resultados Prévios do SR com uso da Métrica de Avaliação}

Foram selecionados 45 docentes da área de computação, do ensino superior, para efetuar a avaliação do SR utilizando as duas sugestões com e sem uso da análise de sentimentos e outros parâmetros (quantidade de curtidas, reações e compartilhamento). Os resultados são mostrados na Tabela 1, utilizando os valores da tabela Likert para avaliação do SR.

Tabela 1. Resultados prévios da avaliação do SR

\begin{tabular}{|c|c|c|}
\hline Valores & $\begin{array}{c}\text { SR sem uso de sentimentos } \\
\text { e outros parâmetros }\end{array}$ & $\begin{array}{c}\text { SR com uso de sentimentos } \\
\text { e outros parâmetros }\end{array}$ \\
\hline Muito bom & $69 \%$ & $89 \%$ \\
\hline Bom & $8 \%$ & $8 \%$ \\
\hline Neutro & $11 \%$ & $2 \%$ \\
\hline Ruim & $9 \%$ & $1 \%$ \\
\hline Muito ruim & $3 \%$ & $0 \%$ \\
\hline
\end{tabular}

\section{Considerações Finais}

Os resultados prévios mostraram a efetividade da utilização do SR com uso da métrica proposta. A análise de sentimentos se mostrou suficiente como parâmetro para a recomendação no contexto educacional. Como trabalhos futuros pretende-se aumentar a quantidade de testes com mais participantes avaliadores e efetuar testes em outras áreas de conhecimento. Pretende-se estudar demais parâmetros que possam auxiliar na recomendação de conteúdo.

\section{Referência}

Almeida, R. F., Campos, F., Ströele, V., David, M. N. (2015). "Sistemas de Recomendação de Recursos Educacionais para Grupos de Redes Sociais: um Mapeamento Sistemático”. In: XXVI SBIE 2015, Cbie-Laclo 2015, p. 1022-1031.

Pang B., and Lee, L. (2008). "Opinion mining and sentiment analysis". Foundations and Trends in Information Retrieval 2(1-2), pp. 1-135.

Pereira, C. K., Campos, F., Ströele, V., N, J. M., Braga, R. (2015). "Explorando Dados Ligados através de um Sistema de Recomendação Educacional". Anais do XXVI Simpósio Brasileiro de Informática Na Educação (SBIE), p. 1042-1051. 